\title{
VALIDACIÓN DE UN MODELO MATEMÁTICO PARA LA CALIBRACIÓN DE FRENÓMETROS PARA MOTOCICLETAS Y PARA VEHÍCULOS LIVIANOS
}

\author{
Paula Raquel Solano Sánchez
}

\begin{abstract}
Resumen
Los frenómetros para motocicletas y vehículos livianos son equipos de medición utilizados para evaluar la eficacia de frenado de las motocicletas y de los vehículos livianos. Este artículo presenta un modelo matemático propuesto para la calibración específica de estos equipos de medición, las fuentes de incertidumbre consideradas para el modelo en cuestión y su cuantificación, así como los principales resultados de la validación de este modelo matemático. Al finalizar el proceso de investigación se concluye que el modelo matemático propuesto es adecuado para la calibración de este tipo de frenómetros, dado que el índice de idoneidad es de 2,2. Por otro lado, al comparar la mejor capacidad de medición del método propuesto con el método utilizado actualmente, se puede observar una mejora del $20 \%$.
\end{abstract}

Palabras clave: metrología, calibración, frenómetros, incertidumbre.

\begin{abstract}
The force measurement equipment used for motorcycles and light vehicles are measuring equipment used to assess the effectiveness of the braking of motorcycles and light vehicles. This article presents a mathematical model for the specific calibration of these measurement equipment. The sources of uncertainty considered for the model and their quantification as well as the main results of the validation of the mathematical model are included.

At the end of the research process it can be concluded that the mathematical model is adequate for the calibration of this type of force measurement equipment given that the suitability is 2,2 .

On the other hand, when you compare the improved capacity of the proposed method, with the method used now, a $20 \%$ improvement is observed.
\end{abstract}

Key words: metrology, calibration, force measurement equipment, uncertainty.

Recibido: 06 de febrero del 2009 • Aprobado: 17 de abril del 2009.

\section{INTRODUCCIÓN}

La inspección técnica vehicular es un conjunto de operaciones realizadas para la verificación del estado general de los vehículos automotores. Esta actividad propicia una cultura de mantenimiento vehicular permanente, incrementa la seguridad al detectar deficiencias de los vehículos producidas por el uso, favorece la reducción del consumo de los combustibles y consigue la reducción de la contaminación que produce el vehículo.

El proceso de inspección vehicular incluye la revisión visual de la estructura del vehículo, tal como el estado de las llantas, el parabrisas, las luces, los cinturones de seguridad, entre otros. También se revisa la alineación de la dirección, la suspensión del vehículo, los frenos y los niveles de contaminantes en el gas de escape.

Para la verificación del estado del sistema de frenos de los vehículos se utiliza un frenómetro. Con él se evalúa la eficacia de frenado; es decir, la relación entre la fuerza total de frenado del vehículo y el peso de este, expresada en porcentaje.

En Costa Rica se utilizan tres tipos de frenómetros de rodillos: frenómetros para motocicletas, frenómetros para vehículos livianos, 
y frenómetros universales. Este artículo presenta un modelo matemático para la calibración de los frenómetros así como para motocicletas y para vehículos livianos y los principales resultados de la validación del mismo.

\section{CARACTERÍSTICAS CONSTRUCTIVAS DE LOS FRENÓMETROS DE RODILLOS}

Un frenómetro de rodillos para vehículos livianos está conformado por dos plataformas que poseen tres rodillos empotrados en el suelo sobre los que se colocan las llantas del vehículo por inspeccionar (Figura 1). Los rodillos (1) y (2) se accionan mediante motores eléctricos que generan una rotación a una velocidad media equivalente a los $5 \mathrm{~km} / \mathrm{h}$. El rodillo (3) cumple la función de sensar la velocidad de rotación de la rueda del vehículo.

La carcasa del motor (4) se encuentra conectada a un transductor extensiométrico (5). Cuando se acciona el sistema de frenado del vehículo, la resistencia al giro de la rueda del vehículo hace que la carcasa gire sobre su propio eje. El transductor censa la variación de resistencia eléctrica que se produce en el puente Wheatstone, que a la vez provoca una diferencia de tensión, en milivolt. Las señales del transductor son transmitidas a una tarjeta de adquisición de datos y un software interpreta estas señales y las convierte en la lectura que se despliega en una pantalla.
El frenómetro para motocicletas funciona igual al frenómetro para vehículos livianos; la diferencia es que el primero solo tiene una plataforma que posee los tres rodillos antes indicados.

\section{EL PRIMER MÉTODO DE CALIBRACIÓN DE FRENÓMETROS UTILIZADO EN COSTA RICA}

En el año 2004, se gesta un proyecto de investigación contratado en el Instituto de Investigaciones en Ingeniería (INII) de la Universidad de Costa Rica (UCR), para desarrollar y validar seis métodos de calibración de equipos de inspección vehicular, entre ellos el método para calibrar los frenómetros para motocicletas, para vehículos livianos y para vehículos pesados.

Este método establece la forma de ejecución y la evaluación de la incertidumbre del proceso de calibración de los frenómetros utilizados para evaluar la eficacia de frenado de motocicletas, de vehículos livianos y de vehículos pesados (Díaz, 2004).

Se calibran tres tipos de frenómetros: los de motocicletas y vehículos livianos en su escala de fuerza; los universales (utilizados para vehículos pesados) en su escala de fuerza y masa. Los patrones a utilizar en el proceso de calibración se citan en el Cuadro 1.

Díaz (2004) señala que para calibrar el frenómetro en su escala de fuerza, se coloca la

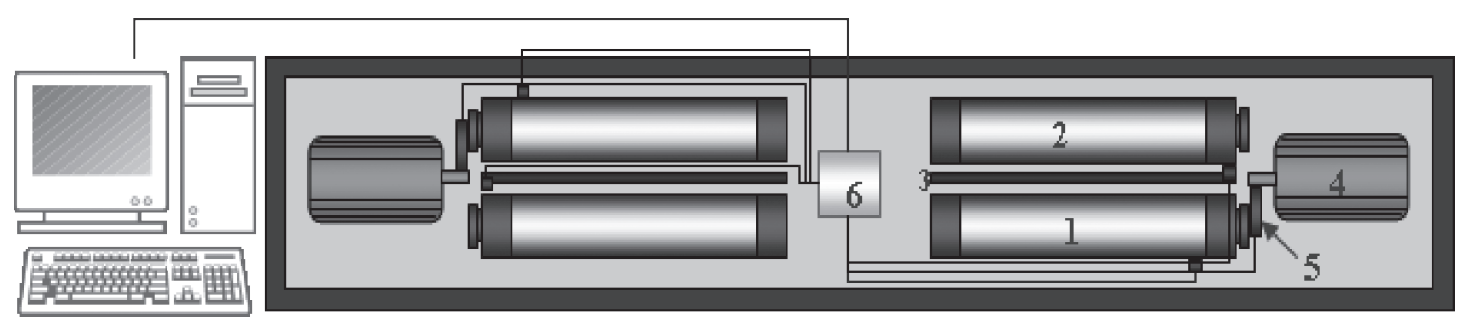

Figura 1. Diagrama de un frenómetro para vehículos livianos.

Fuente: (La autora). 
Cuadro 1. Patrones para calibrar los tres tipos de frenómetros.

Tipo de frenómetro

Patrones

\begin{tabular}{lc}
\hline Escala de fuerza & Escala de masa \\
\hline
\end{tabular}

Para motocicletas y para vehículos livianos

Universales

\author{
Masa de $30 \mathrm{~kg}$, barra de calibración con muescas certificadas en las \\ distancias equivalentes a $0,5 \mathrm{kN}, 1 \mathrm{kN}, 2 \mathrm{kN}, 3 \mathrm{kN}, 4 \mathrm{kN}, 5 \mathrm{kN}$, indicador de \\ temperatura y humedad relativa.
}

Masa de $30 \mathrm{~kg}$, barra de calibración con muescas certificadas en las distancias equivalentes a $3 \mathrm{kN}, 6 \mathrm{kN}, 9 \mathrm{kN}, 12 \mathrm{kN}, 15 \mathrm{kN}$, indicador de temperatura y humedad relativa.

Fuente: (Díaz, 2 004). barra de calibración, de forma horizontal respecto al suelo, y con la barra de contrapeso se asegura la indicación a cero. La masa patrón se coloca en la primera muesca certificada de la barra, simulando un valor de fuerza conocido que se compara con la indicación que muestra la consola del frenómetro. El proceso descrito se realiza en todas las muescas certificadas de la barra de calibración $0,5 \mathrm{kN}$, $1 \mathrm{kN}, 2 \mathrm{kN}, 3 \mathrm{kN}$ para motocicletas; los puntos anteriores, $4 \mathrm{kN}$ y $5 \mathrm{kN}$ para vehículos livianos; $3 \mathrm{kN}, 6 \mathrm{kN}, 9 \mathrm{kN}, 12 \mathrm{kN}, 15 \mathrm{kN}$ para vehículos pesados), en orden ascendente, y se repite pero en sentido descendente. Se registran la temperatura y la humedad relativa del sitio antes y después de la calibración.

Para calibrar el frenómetro en su escala de masa, Díaz (2004) apunta que se registra la indicación de masa del frenómetro libre de peso $\mathrm{y}$, luego, se colocan $250 \mathrm{~kg}$ en masas distribuidas uniformemente. El valor conocido de masa se compara con la indicación que muestra la consola del frenómetro. El proceso descrito se repite para $500 \mathrm{~kg}, 750 \mathrm{~kg}, 1000 \mathrm{~kg}$ y $1250 \mathrm{~kg}$, en orden ascendente, y se repite pero en sentido descendente.

Error de indicación y estimación de la incertidumbre. De acuerdo con Díaz (2004), el mensurando, en la escala de fuerza, es el error de indicación descrito por la ecuación 1.

$\mathrm{e}_{\mathrm{Mj}}=\left(\mathrm{Ic}-\mathrm{M}_{\mathrm{f}}\right)_{\mathrm{j}}=\left(\mathrm{Ic}-10^{-3} \cdot \mathrm{K} \cdot \mathrm{l}_{\mathrm{b}} \cdot \mathrm{m}_{\mathrm{p}}\right)_{\mathrm{j}}$
En donde:

Ic indicación del frenómetro, en $\mathrm{kN}$.

$\mathrm{M}_{\mathrm{f}} \quad$ fuerza de frenado, en $\mathrm{kN}$.

$\mathrm{K}$ factor de conversión que considera entre otros la gravedad local, en $\mathrm{kN}(\mathrm{mm} \bullet \mathrm{kg})^{-1}$.

$l_{\mathrm{b}} \quad$ valor certificado de la barra de calibración, en $\mathrm{mm}$.

$\mathrm{m}_{\mathrm{p}} \quad$ valor certificado de la masa de $30 \mathrm{~kg}$, en $\mathrm{kg}$.

j j-ésimo punto calibrado.

Las fuentes de incertidumbre consideradas en este modelo por Díaz (2004) son las indicadas en el Cuadro, en donde:

Donde:

$\mathrm{u}\left(\mathrm{I}_{\mathrm{C}}\right) \quad$ incertidumbre estándar que aporta el error por resolución, en $\mathrm{kN}$. error por resolución del indicador del frenómetro, en $\mathrm{kN}$.

$\mathrm{u}\left(\mathrm{K}_{\mathrm{b}}\right) \quad$ incertidumbre estándar que aporta el error por trazabilidad de las marcas de longitud de la barra de calibración, en mm.

$\mathrm{u}\left(\mathrm{K}_{\mathrm{m}}\right) \quad$ incertidumbre estándar que aporta el error por trazabilidad de la masa patrón, en $\mathrm{kg}$.

$\mathrm{U}_{\mathrm{p}} \quad$ incertidumbre expandida reportada en el certificado de calibración del patrón, en $\mathrm{mm}$ o g. 
Cuadro 2. Fuentes de incertidumbres consideradas para el modelo de la ecuación (1).

\section{Fuente}

Error por resolución de las mediciones de fuerza en el indicador del frenómetro.

Error por trazabilidad de las marcas de longitud de la barra de calibración.

Error por trazabilidad de la masa patrón.

Error por el efecto de la histéresis mecánica del sistema de medición.
Tipo de evaluación

$\mathrm{B}$, rectangular simétrica

B, normal

B, normal

A, normal
Ecuación

$u\left(I_{C}\right)=\frac{e_{\text {res }}}{\sqrt{3}}$

$\mathrm{u}\left(\mathrm{K}_{\mathrm{b}}\right)=\frac{\mathrm{U}_{\mathrm{p}}}{\mathrm{k}}$

$u\left(K_{m}\right)=\frac{U_{p}}{k}$

$\mathrm{u}\left(\Delta \mathrm{ec}_{\mathrm{hj}}\right)=\frac{\mathrm{w}_{\mathrm{j}}}{\mathrm{D}(\mathrm{n})}$

Fuente: (Díaz, 2004).

$u\left(\Delta e c_{h j}\right) \quad$ incertidumbre estándar que aporta el error por el efecto de la histéresis mecánica, en $\mathrm{kN}$.

$\mathrm{w}_{\mathrm{j}} \quad$ diferencia de las mediciones en ascenso y descenso para el j-ésimo punto calibrado, en $\mathrm{kN}$.

$\mathrm{D}(\mathrm{n}) \quad$ estadística que permite la estimación de la desviación estándar a partir del rango y del número de observaciones.

La incertidumbre combinada que presenta este primer método se estima de acuerdo con la ecuación (2) y la incertidumbre expandida se estima a partir de la ecuación (3).
$\left\{\mathrm{u}^{2}\left(\mathrm{I}_{\mathrm{C}}\right)+\left[\mathrm{c}_{\mathrm{b}} \cdot \mathrm{u}\left(\mathrm{K}_{\mathrm{b}}\right)\right]^{2}+\left[\mathrm{c}_{\mathrm{m}} \cdot \mathrm{u}\left(\mathrm{K}_{\mathrm{m}}\right)\right]^{2}+\mathrm{u}^{2}\left(\Delta \mathrm{e}_{\mathrm{h}}\right)\right\}_{\mathrm{j}}$

En donde:

$\mathrm{u}\left(\mathrm{ec}_{\mathrm{j}}\right) \quad$ incertidumbre combinada, en kN.

$\mathrm{c}_{\mathrm{b}} \quad$ coeficiente de sensibilidad con respecto a la longitud.

$\mathrm{c}_{\mathrm{m}} \quad$ coeficiente de sensibilidad con respecto a la masa.

$$
\mathrm{U}_{\mathrm{pj}}= \pm \mathrm{k}_{\mathrm{p}} \cdot \mathrm{u}\left(\mathrm{ec}_{\mathrm{j}}\right)
$$

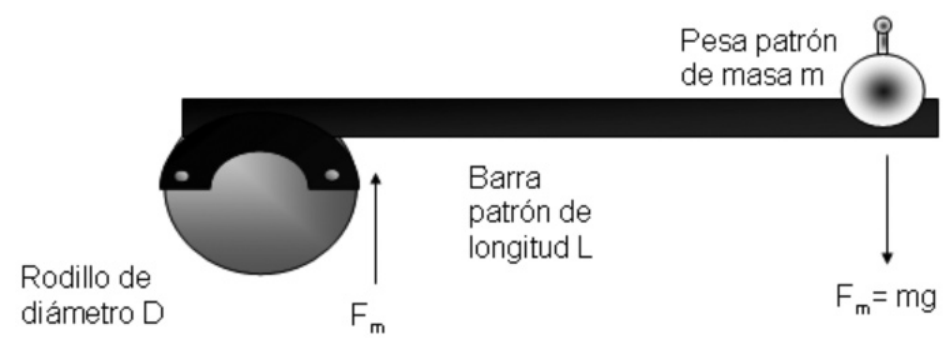

Figura 2. Sistema que genera la fuerza patrón en la calibración de frenómetros para motocicletas y para vehículos livianos.

Fuente: (La autora). 
En donde:

$\mathrm{U}_{\mathrm{pj}} \quad$ incertidumbre expandida, en $\mathrm{kN}$.

$\mathrm{k}_{\mathrm{p}}$ factor de cobertura $\left(\mathrm{k}_{\mathrm{p}}=2\right)$, asociado a un nivel de confianza aproximadamente igual al $95 \%$.

Los parámetros de desempeño reportados para este método son los siguientes:

- Incertidumbre requerida por el proceso de calibración igual a $0,14 \mathrm{kN}$.

- Índice de idoneidad igual a 1,7.

- Mejor capacidad de medición igual a 0,10 $\mathrm{kN}$.

\section{MODELO MATEMÁTICO PROPUESTO PARA LA CALIBRACIÓN DE FRENÓMETROS}

La experiencia generada en el trabajo de campo, desarrollado por LABCAL entre el 2005 y el 2007, llevó al desarrollo de una propuesta para un modelo matemático que describa la calibración de los frenómetros, separando los frenómetros para motocicletas y para vehículos livianos de los frenómetros universales (para vehículos pesados). A continuación se describe el proceso de calibración de frenómetros para motocicletas y para vehículos livianos que da origen al modelo matemático, en su escala de fuerza.

La calibración de los frenómetros para motocicletas y vehículos livianos se realiza mediante la comparación de la indicación del frenómetro con una fuerza patrón generada mediante el sistema que se muestra en la Figura 2. Los patrones a utilizar en el proceso de calibración se citan en el Cuadro 3.

El diámetro del rodillo se mide utilizando el calibrador vernier,el cual debe tener una capacidad de medición de al menos igual al diámetro del rodillo del frenómetro. Se utiliza un promedio de diez mediciones realizadas en al menos dos posiciones angulares. El proceso descrito en este párrafo no se incluye en el método descrito por Díaz (2004).

El frenómetro se calibra en los mismos puntos de calibración del método descrito por Díaz (2004):

- Frenómetros para motocicletas: $0,5 \mathrm{kN}, 1 \mathrm{kN}$, $2 \mathrm{kN}, 3 \mathrm{kN}$ (este último se calibra sólo si el frenómetro lo permite).

- Frenómetros para vehículos livianos: $0,5 \mathrm{kN}$, $1 \mathrm{kN}, 2 \mathrm{kN}, 3 \mathrm{kN}, 4 \mathrm{kN}$ y $5 \mathrm{kN}$.

Y la calibración es independiente para cada sector del frenómetro (derecho e izquierdo).

La reproducción de la fuerza patrón se realiza utilizando el sistema presentado en la Figura 2. Primero, se registra el valor de la indicación del frenómetro sin carga; es decir, 0 $\mathrm{kN}$. Posteriormente, se coloca la masa patrón en las diferentes muescas certificadas de la barra patrón, en orden ascendente y descendente, respectivamente. El proceso se repite en dos ocasiones más. Como se puede notar, el proceso descrito en este párrafo es similar al descrito por Díaz (2004); la variación está dada en la cantidad de repeticiones.

Cuadro 3. Patrones para calibrar los frenómetros para motocicletas y vehículos livianos.

Magnitud

Longitud

Masa
Patrón

Calibrador vernier con capacidad de medición de al menos $250 \mathrm{~mm}$, con exactitud de $0,05 \mathrm{~mm}$.

Barra de calibración con muescas certificadas en las distancias requeridas para reproducir $0,5 \mathrm{kN}$, $1 \mathrm{kN}, 2 \mathrm{kN}, 3 \mathrm{kN}, 4 \mathrm{kN}, 5 \mathrm{kN}$.

Masa de $30 \mathrm{~kg}$ con exactitud de $15 \mathrm{~g}$. 
Cuadro 4. Fuentes de incertidumbres consideradas para el modelo de la ecuación (4).

\section{Fuente}

División de escala del frenómetro

Estabilidad de la lectura del frenómetro

Trazabilidad de la masa patrón

Deriva de la masa patrón

Variación de la gravedad local

Histéresis mecánica del sistema de medición

Trazabilidad de la longitud patrón

Deriva de la longitud patrón

División de escala del vernier

Trazabilidad del calibrador vernier

Variación de la temperatura en la medición del diámetro promedio

r\&R en las mediciones del diámetro

r\&R en las mediciones de fuerza

\section{Tipo de evaluación}

$\mathrm{B}$, rectangular

B, rectangular

$\mathrm{B}$, rectangular

B, rectangular

B, rectangular

$\mathrm{B}$, rectangular

B, normal

$\mathrm{B}$, rectangular

$\mathrm{B}$, rectangular

$\mathrm{B}$, rectangular

B, rectangular

A, normal

A, normal

Fuente: (La autora).

\section{Ecuación}

$$
\mathrm{u}_{\mathrm{MDV}}(\mathrm{Ic})=\frac{\mathrm{d}_{\mathrm{f}}}{2 \sqrt{3}}
$$$$
\mathrm{u}_{\text {est }}(\mathrm{Ic})=\frac{\mathrm{Ic}_{\mathrm{E}_{\max }}-\mathrm{Ic}_{\mathrm{E}_{\min }}}{\sqrt{3}}
$$

$$
u_{\text {traz }}\left(m_{p}\right)=\frac{E_{m_{p}}}{\sqrt{3}}
$$

$$
\mathrm{u}_{\mathrm{der}}\left(\mathrm{m}_{\mathrm{p}}\right)=\frac{\mathrm{x}_{\mathrm{m}_{\mathrm{p}}}}{\sqrt{3}}
$$

$$
\mathrm{u}(\mathrm{g})=\frac{\Delta \mathrm{g}}{\sqrt{3}}
$$

$\mathrm{u}_{\mathrm{h}}\left(\mathrm{ec}_{\mathrm{j}}\right)=\frac{\left|\mathrm{w}_{\mathrm{hmax}}-\mathrm{w}_{\mathrm{hmin}}\right|}{\sqrt{3}}$

$$
\mathrm{u}_{\text {traz }}\left(\mathrm{L}_{\mathrm{p}}\right)=\frac{\mathrm{U}_{\mathrm{L}_{\mathrm{p}}}}{\mathrm{k}}
$$

$$
\mathrm{u}_{\mathrm{der}}\left(\mathrm{L}_{\mathrm{p}}\right)=\frac{\mathrm{x}_{\mathrm{L}_{\mathrm{p}}}}{\sqrt{3}}
$$

$$
u_{M D V}(\bar{D})=\frac{d_{c V}}{2 \sqrt{3}}
$$

$$
\mathrm{u}_{\text {traz }}(\overline{\mathrm{D}})=\frac{\mathrm{E}_{\mathrm{CV}}}{\sqrt{3}}
$$

$$
\mathrm{u}_{\Delta \mathrm{t}}(\overline{\mathrm{D}})=\frac{\mathrm{L}_{0} \alpha \Delta \mathrm{T}}{\sqrt{12}}
$$

$u_{r}^{2}(\bar{D})=s_{R}^{2}(\bar{D})=s_{r}^{2}+\frac{s_{d}^{2}-s_{r}^{2}}{\bar{n}}$

$\mathrm{u}_{\mathrm{r}}^{2}(\overline{\mathrm{Ic}})=\mathrm{s}_{\mathrm{R}}^{2}(\overline{\mathrm{Ic}})=\mathrm{s}_{\mathrm{r}}^{2}+\frac{\mathrm{s}_{\mathrm{d}}^{2}-\mathrm{s}_{\mathrm{r}}^{2}}{\overline{\bar{n}}}$ 
Error de indicación y estimación de la incertidumbre. El modelo matemático que se propone para describir el error de indicación se determina de acuerdo con la ecuación (4).

$\mathrm{ec}_{\mathrm{j}}=\left(\overline{\mathrm{Ic}}-\mathrm{F}_{\mathrm{p}}\right)_{\mathrm{j}}=\left(\overline{\mathrm{Ic}}-2 \times 10^{-3} \cdot \mathrm{m}_{\mathrm{p}} \cdot \mathrm{g} \cdot \frac{\mathrm{L}_{\mathrm{p}}}{\mathrm{D}}\right)$

En donde:

ec error de indicación, en $\mathrm{kN}$.

Ic indicación promedio del frenómetro correspondiente al j-ésimo punto calibrado, en $\mathrm{kN}$.

$\mathrm{F}_{\mathrm{p}} \quad$ valor de fuerza patrón, en $\mathrm{kN}$, y se calcula mediante la ecuación (4).

$\mathrm{m}_{\mathrm{p}} \quad$ masa patrón, en $\mathrm{kg}$.

g aceleración de la gravedad, en $\mathrm{m} / \mathrm{s}^{2}$.

$\mathrm{L}_{\mathrm{p}} \quad$ longitud patrón, en mm.

D diámetro del rodillo, en $\mathrm{mm}$.

j j-ésimo punto calibrado.

Las fuentes de incertidumbre consideradas para este modelo matemático son las descritas en el Cuadro 4 y se calculan siguiendo los lineamientos dados por la Guide to the expression of uncertainty in measurement (BIPM, IEC, IFCC, ISO, IUPAC, IUPAP \& OIML, 1995).

En donde:

$\mathrm{u}_{\mathrm{MDV}}$ (Ic) incertidumbre por la división de escala del frenómetro, en $\mathrm{kN}$.

$\mathrm{d}_{\mathrm{f}} \quad$ mínima división de escala del indicador del frenómetro, en $\mathrm{kN}$.

$\mathrm{u}_{\mathrm{est}}$ (Ic) incertidumbre por la estabilidad del frenómetro.

$\mathrm{Ic}_{\mathrm{E} \max } \quad$ valor máximo de fuerza que indica el frenómetro en la prueba de estabilidad, en $\mathrm{kN}$.

$\mathrm{Ic}_{\mathrm{E} \text { min }} \quad$ valor mínimo de fuerza que indica el frenómetro en la prueba de estabilidad, en $\mathrm{kN}$.

$\mathrm{u}_{\text {traz }}\left(\mathrm{m}_{\mathrm{p}}\right)$ incertidumbre por trazabilidad de la masa patrón, en $\mathrm{kg}$.

$\mathrm{E}_{\mathrm{m}_{\mathrm{p}}} \quad$ exactitud requerida para la masa patrón, en kg. $\mathrm{u}_{\mathrm{tder}}(\mathrm{mp})$ incertidumbre por deriva de la masa patrón, en $\mathrm{kg}$.

$\mathrm{x}_{\mathrm{m}_{\mathrm{p}}} \quad$ deriva de la masa patrón, en $\mathrm{kg}$.

$\mathrm{u}(\mathrm{g}) \quad$ incertidumbre por gravedad local, en $\mathrm{g} / \mathrm{m}^{2}$.

$\Delta \mathrm{g} \quad$ variación de la gravedad local, en $\mathrm{g} / \mathrm{m}^{2}$.

$\mathrm{u}_{\mathrm{h}}\left(\mathrm{ec}_{\mathrm{j}}\right)$ incertidumbre por el efecto de histéresis mecánica en el j-ésimo punto calibrado, en $\mathrm{kN}$.

$\mathrm{w}_{\mathrm{h} \max }$ error de histéresis máximo en el $\mathrm{j}$ ésimo punto calibrado, $\mathrm{kN}$.

$\mathrm{w}_{\mathrm{h} \text { min }}$ error de histéresis mínimo en el $\mathrm{j}$ ésimo punto calibrado, $\mathrm{kN}$.

$\mathrm{u}_{\text {traz }}\left(\mathrm{L}_{\mathrm{p}}\right)$ incertidumbre por trazabilidad de la longitud patrón, en mm.

$\mathrm{U}_{\mathrm{L}_{\mathrm{p}}} \quad$ valor de incertidumbre reportado en el certificado de la barra de patrón, en mm.

k factor de cobertura reportado en el certificado de la barra patrón.

$\mathrm{u}_{\mathrm{tder}}\left(\mathrm{L}_{\mathrm{p}}\right)$ incertidumbre por deriva de la longitud patrón, en mm.

$\mathrm{x}_{\mathrm{L}_{\mathrm{p}}} \quad$ deriva de la longitud patrón, en $\mathrm{mm}$.

$\mathrm{u}_{\mathrm{MDV}}(\overline{\mathrm{D}})$ incertidumbre por la división de escala del calibrador vernier, en $\mathrm{mm}$.

$\mathrm{d}_{\mathrm{cv}} \quad$ mínima división de escala del indicador del calibrador vernier, en mm.

$\mathrm{u}_{\text {traz }}(\overline{\mathrm{D}})$ incertidumbre por trazabilidad del calibrador vernier, en mm.

$\mathrm{E}_{\mathrm{cv}} \quad$ exactitud requerida para el calibrador vernier, en $\mathrm{mm}$.

$\mathrm{u}_{\Delta \mathrm{t}}(\overline{\mathrm{D}})$ incertidumbre por la variación de la temperatura, en $\mathrm{mm}$.

$\Delta \mathrm{T} \quad$ máxima variación de temperatura, en $\mathrm{mm}$.

$\alpha \quad$ coeficiente de expansión térmica , en $\mathrm{mm}$.

$\mathrm{L}_{0} \quad$ diámetro promedio, en $\mathrm{mm}$.

$\mathrm{u}_{\mathrm{r}}^{2}(\overline{\mathrm{D}}) \quad$ incertidumbre por repetibilidad en las indicaciones del calibrador vernier, en $\mathrm{mm}$.

$\mathrm{s}_{\mathrm{R}}^{2}(\overline{\mathrm{D}}) \quad$ varianza de reproducibilidad, obtenida de datos históricos, en mm.

$\mathrm{s}_{\mathrm{r}}^{2} \quad$ varianza por repetibilidad, obtenida de datos históricos, en mm.

varianza entre datos, obtenida de datos históricos, en mm.

promedio de los números de datos promedio. 
Cuadro 5. Requisitos metrológicos del mejor frenómetro calibrado en LABCAL.

Característica

Rango de medición

Mínima división de escala

Exactitud requerida
Valor

De $0 \mathrm{kN}$ a $5 \mathrm{kN}$

$0,01 \mathrm{kN}$

$0,25 \mathrm{kN}$ para el rango de $0 \mathrm{kN}$ a $3 \mathrm{kN}$

$0,50 \mathrm{kN}$ para el rango de $4 \mathrm{kN}$ a $5 \mathrm{kN}$

Fuente: (La autora).

\section{VALIDACIÓN DEL MODELO MATEMÁTICO PROPUESTO}

El proceso de validación se dividió en tres etapas: determinación de los requisitos metrológicos, determinación de los parámetros de desempeño del modelo y evaluación del desempeño.

Etapa 1. Determinación de los requisitos metrológicos. La incertidumbre requerida por el proceso de calibración se definió con base en los requisitos metrológicos del mejor equipo de medición que se calibra en Costa Rica (Cuadro 5).

Etapa 2. Determinación de los parámetros de desempeño del modelo. Se consideró que la selección de los patrones adecuados para la calibración debería garantizar al menos una relación de exactitud mayor o igual a tres. Para determinar los requerimientos de los patrones, se utilizó la ecuación (5).

$$
\mathrm{RE}=\frac{\mathrm{u}_{\mathrm{c}}(\mathrm{y})}{\mathrm{u}_{\mathrm{p}}(\mathrm{y})}
$$

Donde:

RE relación de exactitud, adimensional.

$\mathrm{u}_{\mathrm{p}}(\mathrm{y}) \quad$ incertidumbre estándar asociada a la trazabilidad del patrón, en $\mathrm{kN}$.

$\mathrm{u}_{\mathrm{c}}(\mathrm{y})$ incertidumbre combinada de la medición, en $\mathrm{kN}$.

Nota: se consideró inicialmente que la $\mathrm{u}_{\mathrm{c}}(\mathrm{y})$ es igual a la incertidumbre requerida para el proceso de calibración $\mathrm{u}_{\mathrm{req}}(\mathrm{y})$, la cual fue calculada como un tercio de la exactitud definida para el frenómetro.

Como se observa en el Cuadro 6, las relaciones de exactitud obtenidas para los patrones, descritos en el Cuadro 3, son mucho mayores a tres, por lo que se consideran adecuados para satisfacer los requisitos de la calibración.

Para realizar la estimación de la incertidumbre de la calibración de los frenómetros se utilizó el método descrito por ISO 5725-2. El análisis estadístico se realizó con ocho juegos de datos para cada punto de calibración y para cada sector del frenómetro; y se consideraron las

Cuadro 6. Incertidumbre requerida y relación de exactitudes obtenidas.

Rango de medición

$0 \mathrm{kN}$ a $3 \mathrm{kN}$

$4 \mathrm{kN}$ a $5 \mathrm{kN}$

$$
\mathbf{u}_{\text {req }}(\mathbf{y})
$$

RE

$$
0,08 \mathrm{kN}
$$

$0,17 \mathrm{kN}$
5

11 
Cuadro 7. Incertidumbre combinada de la calibración de un frenómetro liviano

$\begin{array}{ccc}\text { Valor nominal } & \text { Lado izquierdo } & \text { Lado derech } \\ (\mathbf{k N}) & (\mathbf{k N}) & (\mathbf{k N}) \\ 0,00 & 0,036427 & 0,022901 \\ 0,50 & 0,036474 & 0,022921 \\ 1,00 & 0,036613 & 0,022981 \\ 2,00 & 0,037163 & 0,023219 \\ 3,00 & 0,038063 & 0,023610 \\ 4,00 & 0,033055 & 0,025130 \\ 5,00 & 0,034849 & 0,025778\end{array}$

Fuente: (La autora).
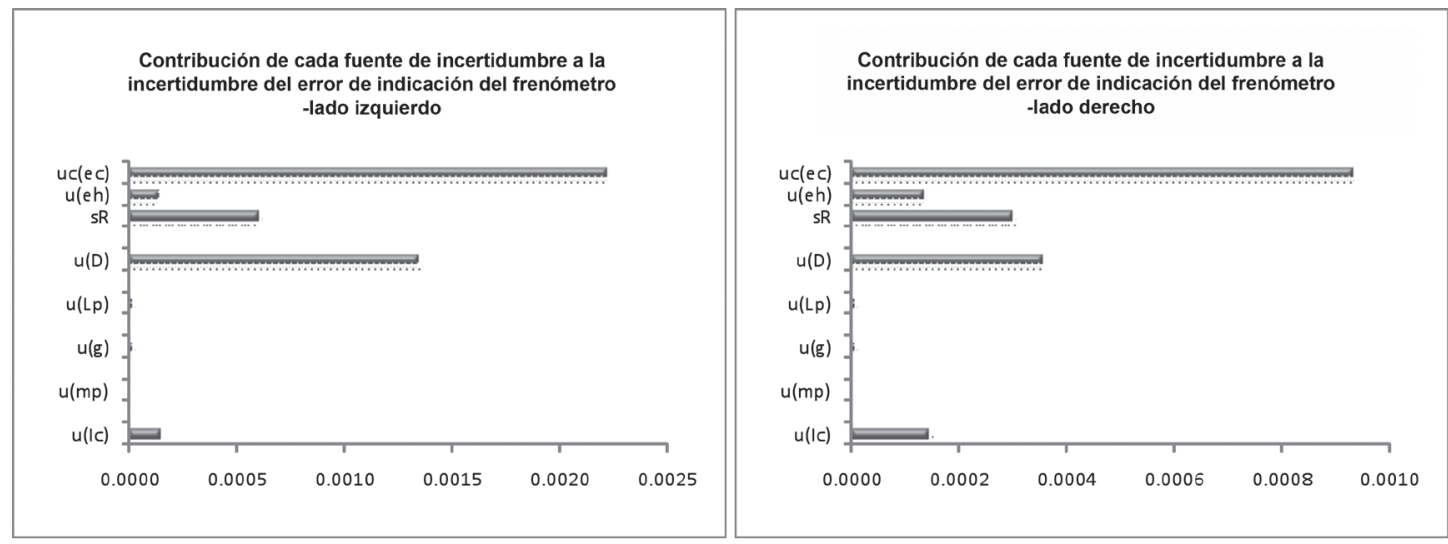

Figura 3. Contribución de las diferentes fuentes de incertidumbre consideradas en el nuevo modelo matemático, para el valor nominal de $5 \mathrm{kN}$.

Fuente: (La autora).

trece fuentes de incertidumbre presentadas en el Cuadro 4.

En el Cuadro 7 se muestran las incertidumbres combinadas, obtenidas para cada punto de calibración. Las fuentes de incertidumbre de más relevancia son asociadas a las mediciones del diámetro del rodillo, a la repetibilidad y reproducibilidad del método, y al efecto de histéresis mecánica del sistema de medición, como se observa en la Figura 3.

Etapa 3. Evaluación del desempeño del modelo. El parámetro utilizado para evaluar el desempeño del método es el índice de idoneidad, descrito por la ecuación (6). El valor óptimo de este índice es igual a uno; sin embargo, se considera que el valor obtenido es satisfactorio para validar el modelo si el índice es mayor o igual a uno.

$\mathrm{I}_{\mathrm{id}}=\frac{\mathrm{u}_{\text {req }}(\mathrm{y})}{\mathrm{u}_{\mathrm{c}}(\mathrm{y})}$

Donde: 


\section{$\mathrm{u}_{\mathrm{req}}(\mathrm{y})$ incertidumbre requerida para el proceso de calibración, en $\mathrm{kN}$. Estos datos se reportan en el Cuadro 6. \\ $\mathrm{I}_{i d} \quad$ índice de idoneidad, adimensional. \\ $\mathrm{u}_{\mathrm{c}}(\mathrm{y})$ incertidumbre combinada de la medición, en kN.}

Para el modelo descrito en la ecuación (4), el mejor índice de idoneidad obtenido es de 2,2; es decir que la incertidumbre requerida es 2,2 veces la incertidumbre combinada. Por lo tanto, este modelo es adecuado para la calibración de frenómetros para motocicletas y para vehículos livianos, en un rango de medición de $0 \mathrm{kN}$ a $5 \mathrm{kN}$, con una mínima división de escala de $0,01 \mathrm{kN}$ y una exactitud requerida de $0,25 \mathrm{kN}$.

Conocido el resultado de la validación, se procedió a calcular la mejor capacidad de medición (MCM), obtenida como la incertidumbre expandida del resultado de la calibración. La MCM obtenida es de $0,08 \mathrm{kN}$, para un factor de cobertura de $\mathrm{k}=2 \mathrm{y}$ un nivel de confianza del $95 \%$.

\section{CONCLUSIONES}

1. El nuevo modelo matemático propuesto para la calibración de frenómetros para motocicletas y para vehículos livianos es adecuado para el uso previsto, dado que cumple con el parámetro de evaluación del desempeño: índice de idoneidad mayor a uno.

2. Para el nuevo modelo matemático fueron cuantificadas trece fuentes de incertidumbre y luego de ser evaluadas se concluye que más del $90 \%$ de la incertidumbre estándar combinada lo aportan las incertidumbres asociadas a las mediciones del diámetro del rodillo, a la repetibilidad y reproducibilidad del método, y al efecto de histéresis mecánica del sistema de medición.

3. Al comparar las mejores capacidades de medición, MCM, se puede observar que la MCM del nuevo modelo matemático mejora en un $20 \%$ con respecto a la obtenida en el modelo descrito por (Díaz, 2004).

\section{NOTAS}

1. Este procedimiento es utilizado como referencia bibliográfica con la autorización de RITEVE SYC, S. A.

\section{REFERENCIAS BIBLIOGRÁFICAS}

Díaz, A. (2004). Calibración del frenómetro. En: Desarrolloy validación de los procedimientos de calibración de los sistemas de medición de RITEVE SYC, S. A. Universidad de Costa Rica .

INTECO. (2006). INTE-ISO 5725-2:2006, Exactitud (veracidad y precisión) de resultados y métodos de precisión - Parte 2: método básico para la determinación de la repetibilidad y reproducibilidad de un método de medición normalizado. Primera edición. San José: Editor.

BIPM, IEC, IFCC, ISO, IUPAC, IUPAP \& OIML. (1995). Guide to the expression of uncertainty in measurement. First edition. InternationalOrganizationforStandarization: Switzerland.

\section{SOBRE LA AUTORA}

\section{Paula Solano Sánchez}

Licenciatura en Ingeniera Química de la Universidad de Costa Rica.

Coordinadora e investigadora del Laboratorio de Metrología, Normalización y Calidad de la Universidad de Costa Rica

Ciudad Universitaria Rodrigo Facio Brenes, San José. Costa Rica

Teléfonos: (506) 2511-5553

Facsímil: (506) 2253-4614

Apartado postal: 11501-2060Correoelectrónico: paulas@inii.ucr.ac.cr. 